\section{BET-ting on bromodomains}

\section{By Joanne Kotz, Senior Editor}

Although histone deacetylases are well-trodden, validated targets, ${ }^{1}$ the therapeutic potential of inhibiting other aspects of the histone acetylation machinery has remained an open question. Now, a team led by the Dana-Farber Cancer Institute and the Structural Genomics Consortium has identified a selective inhibitor of bromodomains-protein domains that bind and recognize histone acetylation - and has shown its activity in a preclinical model of a rare cancer.

Addition of an acetyl group to lysine residues on histones is an epigenetic mark associated with gene activation. These acetyl groups are reversibly maintained by histone acetyltransferases (HATs) and histone deacetylases (HDACs). ${ }^{2}$

Ultimately, histone acetylation is monitored by bromodomaincontaining proteins to regulate epigenetically controlled processes including chromatin remodeling and gene transcription.

There are six families of bromodomain-containing proteins. Research over the last decade has shown that members of one such family - the BET family, which includes the proteins bromodomain containing 2 (BRD2), BRD3 and BRD4-may be potential cancer targets.

For example, researchers at Brigham and Women's Hospital showed in 2003 that NUT midline carcinoma is driven by a chromosomal translocation that fuses the two bromodomains of BRD4 with a protein called nuclear protein in testis (NUT). ${ }^{3}$

The BRD4-NUT fusion is believed to cause cancer by inhibiting the differentiation of the epithelial cells in which it is expressed and thus maintaining them in an undifferentiated and hyperproliferative state. NUT midline carcinoma is always fatal.

To further investigate the role of bromodomains in cancer, a team led by James Bradner and Stefan Knapp decided to develop inhibitors of the BET family of bromodomains. ${ }^{4}$

Bradner is an investigator in the Department of Medical Oncology and a staff physician in the Stem Cell Transplantation and Hematologic Malignancies Services at Dana-Farber and an instructor in the Department of Medicine at Harvard Medical School. Knapp is a principal investigator in the Phosphorylation Dependent Signaling Group of the Structural Genomics Consortium (SGC) Oxford and a professor of structural chemistry in the Nuffield Department of Clinical Medicine at the University of Oxford.

The researchers didn't have to start from scratch. Instead, they designed the lead inhibitor, JQ1, based on information in the scientific literature and a patent held by Mitsubishi Tanabe Pharma Corp. covering thienodiazepines that bind to BRD4.

In vitro, JQ1 bound selectively to bromodomains of the BET protein family and showed little or no detectable binding to 33 other bromodomains spanning the 5 other bromodomain-containing families. Compared with a control molecule, JQ1 inhibited the binding of acetylated histone peptides to BRD4.

In cancer cells from NUT midline carcinoma patients, JQ1 decreased cell proliferation and the expression of BRD4 target genes and increased cellular differentiation compared with a control compound.

Finally, in mouse xenograft models of the cancer, JQ1 increased both tumor cell differentiation and survival and decreased tumor size compared with vehicle control $(p \leq 0.0001)$.

Data were published in Nature.

"Most people in pharma would have dismissed bromodomains as interesting but not druggable" because potent and selective small molecule inhibitors had not been previously identified, said Robert Copeland, EVP and CSO of Epizyme Inc. "This paper proves this conventional wisdom wrong."

Epizyme develops inhibitors of epigenetic enzymes, in particular of histone methyltransferases.

"This paper represents a major development in the field of epigenetics, illustrating for the first time that the interaction between bromodomain epigenetic mark readers and their acetyl-lysine recognition motifs can be blocked by small, drug-like molecules," said Mark Bunnage, executive director of worldwide medicinal chemistry at Pfizer Inc.

\section{Cracking a tough NUT}

Bradner's laboratory now is focused on medicinal chemistry optimization of JQ1 and pharmacokinetic and safety testing of JQ1 derivatives.

"The obvious immediate impact of this work would be in patients with NUT midline carcinoma," said Stephen Frye, professor of medicinal chemistry and natural products and director of the Center for Integrative Chemical Biology and Drug Discovery at The University of North Carolina at Chapel Hill.

Frye, who previously was worldwide VP of discovery medicinal chemistry for GlaxoSmithKline plc, told SciBX that the available pharmacological and pharmacokinetic data on JQ1 suggest it will be a viable starting point for optimization of the series to yield a drug candidate.

Added Bunnage, "Whether JQ1 itself has the appropriate safety and pharmacokinetic profile for clinical studies remains to be seen. There is clear scope, however, for further medicinal chemistry optimization within this inhibitor class."

Robert Sims, director of biology at epigenetics company Constellation Pharmaceuticals Inc., also thinks the inhibitors described in Nature have potential for BRD4-dependent cancers. "The proposed mechanism of action of these inhibitors, induction of differentiation, is 
an atypical yet very exciting avenue for cancer therapy," he said. "More work is needed to confirm that these BET inhibitors indeed function through this mechanism."

In addition to questions about mechanism, Sims said there could be toxicity issues. "Considering the importance of BRD4 in normal cellular function, much more work is needed to characterize the on-target toxicity profile of these compounds in long-term studies," he said.

Because JQ1 was rationally designed, Copeland suggested running a high throughput screen to identify BRD4 inhibitors with other chemical scaffolds. Starting from a range of chemical classes would increase the chances of identifying a pharmacologically tractable lead molecule and avoiding any potential IP issues associated with the JQ1 scaffold, he said.

\section{Upping the ante}

NUT midline carcinoma is a rare cancer that affects only 20-40 patients per year in the U.S. Thus, a key question is whether bromodomain inhibitors will have broader applications.

Bradner and Knapp are now focused on testing JQ1 in preclinical models of other cancers in which BET-family bromodomains are potentially involved. They also are using JQ1 as a starting point to identify inhibitors of other bromodomain-containing protein families implicated in cancer.

Bunnage expects that bromodomain inhibitors, like HDAC inhibitors, will have utility outside of cancer. "In addition to the potential utility in NUT midline carcinoma, there is also literature evidence that the BET family of bromodomains may have therapeutic potential in other areas, including inflammatory diseases," he said.

Sims cautioned that it is not yet clear whether inhibiting proteins with bromodomains will have therapeutic benefit. "Large chromatin remodeling complexes that harbor bromodomains typically contain multiple histone-binding contacts. Will inhibiting a histone-bromodomain interaction result in a loss of function in this setting?"

For the BRD4-NUT-dependent cancer, this appears to be true, said Sims, but "much more work is needed before we understand how broadly this concept applies to other bromodomain-dependent disease states."

Dana-Farber has filed for a patent covering JQ1 derivatives. The IP is available for licensing.

Mitsubishi Tanabe told SciBX it does not have a bromodomain inhibitor in its pipeline.

Kotz, J. SciBX 3(41); doi:10.1038/scibx.2010.1224

Published online Oct. 21, 2010

\section{REFERENCES}

1. Kelly, T.K. et al. Nat. Biotechnol. 28, 1069-1078 (2010)

2. Kouzarides, T. Cell 128, 693-705 (2007)

3. French, C.A. et al. Am. J. Pathol. 159, 1987-1992 (2001)

4. Filippakopoulos, P. et al. Nature; published online Sept. 24, 2010; doi:10.1038/nature09504

Contact: James E. Bradner, Dana-Farber Cancer Institute, Boston, Mass.

e-mail: james_bradner@dfci.havard.edu

Contact: Stefan Knapp, Structural Genomics Consortium, Oxford, U.K. e-mail: stefan.knapp@sgc.ox.ac.uk

\section{COMPANIES AND INSTITUTIONS MENTIONED}

Brigham and Women's Hospital, Boston, Mass.

Constellation Pharmaceuticals Inc., Cambridge, Mass.

Dana-Farber Cancer Institute, Boston, Mass.

Epizyme Inc., Cambridge, Mass.

GlaxoSmithKline plc (LSE:GSK; NYSE:GSK), London, U.K.

Harvard Medical School, Boston, Mass.

Mitsubishi Tanabe Pharma Corp. (Tokyo:4508; Osaka:4508),

Osaka, Japan

Pfizer Inc. (NYSE:PFE), New York, N.Y.

Structural Genomics Consortium, Oxford, U.K.

The University of North Carolina at Chapel Hill, Chapel Hill, N.C. University of Oxford, Oxford, U.K. 